\title{
Homeobox B9 facilitates hypertrophic scar formation via activating the mitogen-activated protein kinase signaling pathway
}

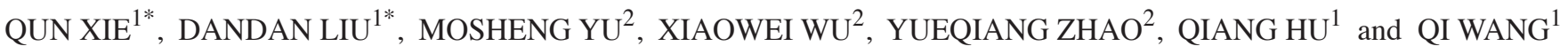 \\ ${ }^{1}$ Department of Plastic Surgery, Xiangyang Central Hospital, Xiangyang, Hubei 441021; \\ ${ }^{2}$ Department of Plastic Surgery, Renmin Hospital of Wuhan University, Wuhan, Hubei 430000, P.R. China
}

Received March 14, 2016; Accepted February 16, 2017

DOI: $10.3892 / \mathrm{mmr} .2017 .6836$

\begin{abstract}
The functions and underlying mechanisms of homeobox B9 (HOXB9) in scar formation remain unclear; therefore, the present study aimed to investigate whether HOXB9 is highly expressed in hypertrophic scar formation. Immunohistochemistry was performed to examine the expression levels of laminin, fibronectin (FN), collagen type I (Col1) and HOXB9 in hypertrophic scar and healthy skin tissues, and in lentivirus-constructed HOXB9-overexpressed or -silenced fibroblasts (FBs). Reverse transcription-quantitative polymerase chain reaction and western blotting were performed to evaluate the mRNA and protein expression levels of HOXB9, laminin, FN, Col1, extracellular signal-regulated kinase (ERK), c-Jun N-terminal kinase (JNK), p38, p-c-Jun N-terminal kinase (JNK), p-ERK and p-p38. A gel contraction assay was used to evaluate the effect of HOXB9 on FB contraction. Co-immunoprecipitation assays were performed to verify the reciprocal interactions between HOXB9 and ERK, JNK and p38. It was demonstrated that HOXB9, laminin, FN and Coll were upregulated in hypertrophic scar tissues, and HOXB9 upregulated laminin, FN, Col1, p-ERK, p-JNK and p38, potentially by interacting directly with p38. Furthermore, FBs overexpressing HOXB9 exhibited enhanced contractile capacity. In conclusion, the present study demonstrated that HOXB9 may facilitate hypertrophic scar formation via activating the mitogen-activated protein kinase signaling pathway.
\end{abstract}

\section{Introduction}

Hypertrophic scars are defined as visible and elevated scars without spreading into surrounding tissues and with a tendency

Correspondence to: Dr Qi Wang, Department of Plastic Surgery, Xiangyang Central Hospital, 163 Jingzhou Road, Xiangcheng, Xiangyang, Hubei 441021, P.R. China

E-mail: 13871771688@163.com

\section{${ }^{*}$ Contributed equally}

Key words: homeobox B9, hypertrophic scar, mitogen-activated protein kinase signaling pathway to regress spontaneously. They are characterized by proliferation of the dermal tissue, with excessive deposition of fibroblast (FB)-derived extracellular matrix (ECM) proteins, particularly collagen, over long periods, and by persistent inflammation and fibrosis $(1,2)$.

Numerous treatments have been described, including surgical excision, pressure therapy, intralesional interferon, laser therapy and silicone gel sheeting (3-7). However, no optimal treatment method has been established, primarily due to limited understanding of the precise underlying mechanisms. Abnormal activation of FBs and accumulation of collagen collaborate to induce hypertrophic scar formation (8). The ECM is primarily derived from FBs, and its activation is considered to facilitate re-epithelialization (9). Furthermore, reduced function of FBs reduces ECM production and leads to cell apoptosis, leading to maturation of the scar (10). The balance between pro- and anti-fibrotic activity is critical to normotrophic scar formation, and failing to regulate activated FBs leads to pathologic scar formation, including hypertrophic scars. Therefore, identifying molecules that strengthen or debilitate may have therapeutic value for the treatment of hypertrophic scars.

Homeobox (HOX) genes encode a group of transcription factors that bind to specific DNA strands via the homeodomain (11). A total of 39 genes are classified into four clusters: HOXA, B, C and D (12). HOXD3 and HOXA3 accelerate wound closure in poorly healing diabetic mice, with improved angiogenesis $(13,14)$. In contrast to HOXA3 and HOXD3, HOXB13 was demonstrated to impair wound healing $(15,16)$. These studies have associated HOX genes with wound healing, an essential process in scar formation, indicating that HOX genes are potentially involved in hypertrophic scar formation. However, to the best of the authors' knowledge, no previous studies have investigated this association.

HOXB9 is a widely understood to be involved in the development of mammary glands, sternum and angiogenesis $(17,18)$. Previous studies have revealed that HOXB9 is involved in the breast cancer, lung adenocarcinoma and gastric carcinoma, serving a role in promoting or inhibiting the tumor process (18-24).

HOXB9 may have an effect on dermal FBs, and facilitate or attenuate hypertrophic scar formation in vivo. Therefore, the present study examined its expression in hypertrophic scar tissues, and tested its effects on contraction. This study further 
investigated the potential biochemical mechanisms involved in the effects of HOXB9 on hypertrophic formation.

\section{Materials and methods}

Ethics statement. All experimental procedures were conducted under a protocol approved by the Ethical Committee of Xiangyang Central Hospital (Xiangyang, China).

Cell culture and treatment. Six patients (2 males and 4 females) were enrolled from March-May 2016 in the Plastic Surgery Department of Xiangyang Central Hospital. The age of the patients ranged 16-40. Hypertrophic scar tissue from the arm and adjacent healthy skin samples were collected from the patients prior to surgical treatment. The tissue was fixed in $4 \%$ formalin. After fixation, the tissue was embedded with paraffin wax. Written consent was obtained from the patients themselves or their legal guardians. Dermal FBs were isolated and cultured as described previously (25). Briefly, tissues were trimmed to remove excessive adipose and rinsed with PBS three times. Next, tissues were sectioned into small pieces and incubated in Dulbecco's Modified Eagle's medium (DMEM; Gibco; Thermo Fisher Scientific, Inc., Waltham, MA, USA) containing 0.1\% collagenase type I (Sigma-Aldrich; Merck KGaA, Darmstadt, Germany) at $37^{\circ} \mathrm{C}$ for $3 \mathrm{~h}$. The isolated FBs were subsequently cultured in DMEM containing 10\% fetal calf serum (Gibco; Thermo Fisher Scientific, Inc.) at $37^{\circ} \mathrm{C}$ in a humidified atmosphere of $5 \% \mathrm{CO}_{2}$. All cells used in this experiment were at passage 5-10. Several 60-mm dishes of healthy skin FBs were randomly divided into different groups $(\mathrm{n}=6)$.

Immunohistochemistry. Paraffin-embedded scar tissues and autologous skin tissues were cut into $5-\mu \mathrm{m}$ thick sections for immunohistochemical staining. Sections were deparaffinized, dehydrated and subject to antigen retrieval by pretreating with $7 \% \mathrm{H}_{2} \mathrm{O}_{2}$ in distilled water, followed by $0.1 \mathrm{~mol} / 1$ periodic acid, $0.005 \mathrm{~mol} / 1 \mathrm{NaBH} 4$ and normal human serum (Thermo Fisher Scientific, Inc.). The sections were separately incubated for $2 \mathrm{~h}$ with rabbit anti-human fibronectin (FN; 1:1,000; catalog no. ab61214; Abcam, Cambridge, MA, USA), rabbit anti-human collagen type I (Col1; 1:200; catalog no. ABIN2473035; Antibodies-Online, Inc., Atlanta, GA, USA), rabbit anti-human laminin (1:300; catalog no. ab11575, Abcam) and rabbit anti-human HOXB9 (1:500; catalog no. ABIN952780; Antibodies-Online, Inc.) at room temperature overnight. After washing with PBS, Envision+/HPR against rabbit (GK400305; Dako; Agilent Technologies, Inc., Santa Clara, CA, USA) was added and incubated for $30 \mathrm{~min}$, followed by detection with 3,3'-diaminobenzidine detection (Sigma-Aldrich; Thermo Fisher Scientific, Inc.). Slides were counterstained with hematoxylin. The sections were washed in water, mounted and observed under a Zeiss Axiophot microscope (Carl Zeiss AG, Oberkochen, Germany). The images were obtained and analyzed using an Image-Pro plus software version 6.0 system (Media Cybernetics, Inc., Rockville, MD, USA).

Establishment of stable HOXB9 overexpression and knockdown in human FBs. A shorthairpin(sh)RNA with high HOXB9 knockdown efficiency was used [HOXB9 small interfering (si)
RNA1: CCCUUCAAUUUGUAGACUCUU], and a shRNA with no effect on HOXB9 levels was used as a control (Control siRNA: UUCUCCGAACGUGUCACGU). As described previously (26), the lentivirus was made using a three-plasmid packaging system (catalog no. 632455; Takara Biotechnology Co., Ltd., Dalian, China). The lentiviral vector system had three parts before packaging, including a pSIREN-RetroQ-ZsGreen1 vector, a pHelper 1.0 (gag/pol element) vector, and a pHelper 2.0 (VSVG element) vector. shRNAs targeting HOXB9 and the negative control were harbored in the pSIREN-RetroQ-ZsGreen1 vector.DNA sequencing confirmed the accurate insertion of the HOXB9shRNA. The pHelper 1.0 plasmid $(15 \mu \mathrm{g})$, the pHelper 2.0 plasmid $(10 \mu \mathrm{g})$ and the pSIREN-RetroQ-ZsGreen 1-HOXB9shRNA plasmid (Clontech Laboratories, Inc., Mountainview, CA, USA) $(20 \mu \mathrm{g})$ were cotransfected into subconfluent 293T cells in serum-free medium using a cationic liposome based transfection reagent (Lipofectamine ${ }^{\circledR}$ 2000; Thermo Fisher Scientific, Inc.). Following $10 \mathrm{~h}$ of incubation, the medium was replaced. Recombinant deficient lentiviruses with HOXB9shRNA were harvested $72 \mathrm{~h}$ later. The FBs were transfected with these recombinant deficient lentiviruses and the clones with green fluorescence were selected under fluorescence microscopy, following which the experimental procedures were performed. Meanwhile, a pLKO.1-puro vector (Addgene, Inc., Cambridge, MA, USA), harboring the HOXB9 cDNA (NCBI reference sequence ID, AH010085) lentivirus was harvested $72 \mathrm{~h}$ after transfection, and $5 \mathrm{mg} / \mathrm{ml}$ polybrene was added. Subconfluent FBs were infected with the harvested lentivirus, and were selected in $2 \mathrm{mg} / \mathrm{ml}$ puromycin. Individual colonies of stable overexpression of HOXB9 and control colonies were isolated and DNA sequencing confirmed the accurate insertion of the HOXB9 cDNA.

Reverse transcription-quantitative polymerase chain reaction $(R T-q P C R)$. Total RNA was isolated from FBs using TRIzol ${ }^{\circledR}$ reagent (Bio-Rad Laboratories, Inc., Hercules, CA, USA) according to the manufacturer's protocol. The RNA samples were treated with RNase-free DNase (Roche Applied Science, Mannheim, Germany) at $37^{\circ} \mathrm{C}$ for $30 \mathrm{~min}$, followed by phenolchloroform extraction and ethanol precipitation. Total RNA $(1.2 \mu \mathrm{g})$ was used in the reverse transcription reaction with oligodT primers. Samples were amplified in an RT-PCR System (Applied Biosystems; Thermo Fisher Scientific, Inc.) using the following conditions: Initial denaturation at $94^{\circ} \mathrm{C}$ for $30 \mathrm{sec}$, followed by 30 cycles denaturation at $95^{\circ} \mathrm{C}$ for $30 \mathrm{sec}$, annealing at $55^{\circ} \mathrm{C}$ for $30 \mathrm{sec}$, and extension at $72^{\circ} \mathrm{C}$ for $30 \mathrm{sec}$ followed by $6 \mathrm{~min}$ at $72^{\circ} \mathrm{C}$. The final relative expressions of mRNA were compared using the $2^{-\Delta \Delta C q}$ method as previously described (27). The primers used were as follows: GAPDH forward, 5'-CTGCCGCTCCATCGTGGCTAG-3' and reverse, 5'-GCCATGCAATGAAT-3' for GAPDH (107 bp band product); forward, 5'-CGCTGGTTCTAAAACTGA GTTTCTG-3' and reverse, 5'-ATCCTGAGAAGGGCGGTG ATC-3' for laminin (117 bp band product); forward, 5'-CCT GTCCTCTTTTCTCGTCTAATC-3'; and reverse, 5'-CTT CTCTTGGAACCAGCGTCTGG-3' for HOXB9 (126 bp band product); forward, 5'-GCTCCATCCAACTGGCGT GTCTGATCC-3' and reverse, 5'-AAGACGAACCCCGTG GCATGT-3' for HOXB9 (186 bp band product); and forward, 
5'-AGCGAAGATGGTTCACTGGGCTCCA-3' and reverse, 5'-GACTTGTCTCTCCTCTAGGAATCC-3' for Col1 (196 bp band product).

Western blot analysis. For western blot cell lysates were prepared using radioimmunoprecipitation assay buffer $(50 \mathrm{mM}$ Tris-HCl, pH 7.4), followed by sonication five times for $10 \mathrm{sec}$ on ice. The lysate was transferred to a microcentrifuge tube and centrifuged at 14,000 $\mathrm{x}$ g for $15 \mathrm{~min}$ to pellet the cell debris. The supernatant containing the protein was retained. Proteins $(30 \mu \mathrm{g})$ were separated on a $12 \%$ denaturing polyacrylamide gel and electro-transferred to a polyvinylidene difluoride membrane (Merck KGaA). The membranes were blocked with TBS with $0.1 \%$ Tween- 20 containing $5 \%$ fat-free dry milk, and subsequently incubated with a primary antibody overnight at $4^{\circ} \mathrm{C}$. The following rabbit anti-human primary antibodies were used: FN (1:1,000; catalog no. ab61214; Abcam), Col1 (1:5,000; catalog no. ABIN2473035; Antibodies-Online, Inc.), extracellular signal-regulated kinase (ERK) 1/2 (1:2,000; catalog no. ab196883; Abcam), phosphorylated (p)-ERK1/2 (1:1,000, catalog no. 9101, Cell Signaling Technology, Inc., Danvers, MA, USA), c-Jun N-terminal kinase (JNK; 1:1,000; catalog no. sc-572; Santa Cruz Biotechnology, Inc., Dallas, TX, USA), p-JNK (1:1,000; catalog no. 4668; Cell Signaling Technology, Inc.), p38 (1:200; catalog no. ab7952; Abcam), p-p38 (1:1,000, catalog no. 9211, Cell Signaling Technology, Inc.), HOXB9 (1:500; catalog no. ABIN952780; Antibodies-Online, Inc.) and GAPDH (1:5,000; catalog no. 2-RGM2; Advanced ImmunoChemical Inc., Long Beach, CA, USA). Following this, membranes were incubated for $1 \mathrm{~h}$ at room temperature with corresponding horseradish peroxidase-conjugated anti-rabbit secondary antibodies (1:2,000; catalog no. sc-2004; Santa Cruz Biotechnology, Inc.) and developed using enhanced chemiluminescence (catalog no. 34077; Thermo Fisher Scientific, Inc.).

Gel contraction assay. The contraction capacity of FBs was detected using the cell contraction assay kit (CBA-201; Cell Biolabs, Inc., San Diego, CA, USA). FB-embedded collagen gels were prepared as described previously $(28,29)$. Briefly, four 24-well plates were pre-treated with $0.4 \%$ bovine serum albumin (Sigma-Aldrich; Merck KGaA) for 2 h. A 0.5 -ml suspension containing $1 \times 10^{6}$ FBs (FB-mock), FBs infected with an empty lentivirus (FB-negative), and FB-HOXB9-overexpressing (FB-HOXB9over) or FB-HOXB9-silenced (FB-HOXB9si) cells, and $2 \mathrm{mg} / \mathrm{ml}$ collagen, were added into the wells. Following this, FB-mock, FB-negative, FB-HOXB9over and FB-HOXB9si cells were incubated at $37^{\circ} \mathrm{C}$ for $48 \mathrm{~h}$ for polymerization, followed by mechanical detachment from the sides of the wells. Gels were imaged at 0,12, 24,36 and $48 \mathrm{~h}$, and the images were analyzed using Image-Pro Plus software, version 6.0.

Co-immunoprecipitation (co-IP). Transfected cells were lysed in cell lysis buffer $(50 \mathrm{mM}$ Tris-HCl, $\mathrm{pH} 8.0 ; 150 \mathrm{mM}$ $\mathrm{NaCl}, 1 \mathrm{mM}$ EDTA, $1 \%$ Nonidet P-40, 10\% glycerol with protease inhibitor mixture) for $1 \mathrm{~h}$. Whole cell extracts were collected and precleared. The beads coated with antibodies were incubated with the precleared whole cell extracts at $4^{\circ} \mathrm{C}$ overnight. The beads were washed with cell lysis buffer four times, following which beads were removed by low speed centrifugation $(2,000 \mathrm{x} \mathrm{g})$ at room temperature for $1 \mathrm{~min}$. Precleared supernatants incubated with anti-HOXB9, ERK, JNK or p38 antibodies for $2 \mathrm{~h}$ in the presence of Protein A-agarose beads. Immunoprecipitated material was collected on the beads, washed extensively and purified proteins were eluted in SDS-PAGE sample buffer. Precipitated proteins were analyzed by western blotting, as described above.

Statistical analysis. To determine significant differences between groups, one-way analysis of variance followed by Bonferroni's post hoc test was performed. The statistical analyses were performed using SPSS software version 20.0 (IBM SPSS, Armonk, NY, USA). GraphPad Prism software version 6.0 (GraphPad Software, Inc., La Jolla, CA, USA) was employed to produce the majority of figures. Data are presented as the mean \pm standard deviation. $\mathrm{P}<0.05$ was considered to indicate a statistically significant difference.

\section{Results}

Expression levels of Coll, FN, laminin and HOXB9 are elevated in hypertrophic scar tissues compared with healthy skin tissues. The ECM consists of numerous components, including collagen, FN and laminin. Previous studies have demonstrated that the ECM is more abundant in hypertrophic scar tissue than that in healthy skin (30-32). The present study tested the expression of Col1, FN, laminin and HOXB9 in hypertrophic scar and healthy skin tissues. These four proteins were elevated in the hypertrophic scar tissues compared with corresponding healthy skin tissues in six patients $(\mathrm{P}<0.05)$, suggesting that the samples selected were in accordance with the pathological standard of hypertrophic scar tissue (Fig. 1).

Expression of Coll, FN and laminin are associated with HOXB9. A lentiviral system was used to overexpress or silence HOXB9 in FBs (FB-HOXB9over and FB-HOXB9si, respectively). Compared with the FB-mock group, mRNA and protein expression levels of HOXB9 were downregulated in FB-HOXB9si cells; whereas they were upregulated in FB-HOXB9over cells (Fig. 2A). Laminin protein expression decreased by $60.33 \pm 3.25 \%$ in the FB-HOXB9si group and increased by $4.12 \pm 0.33$ fold in the FB-HOXB9over group, compared with the FB-mock group $(\mathrm{P}<0.01$; Fig. 2B). FN protein expression levels decreased by $52.27 \pm 4.33 \%$ in the FB-HOXB9si group and increased by $1.18 \pm 0.23$-fold in the FB-HOXB9over group, compared with the FB-mock group $(\mathrm{P}<0.01$; Fig. 2C). Col1 protein expression levels decreased by $44.53 \pm 2.85 \%$ in the FB-HOXB9si group and increased by $2.12 \pm 0.37$-fold FB-HOXB9over group, compared with the FB-mock group $(\mathrm{P}<0.01$; Fig. 2D). mRNA expression levels of laminin and Col1 were consistent with the corresponding protein expression levels $(\mathrm{P}<0.01$; Fig. $2 \mathrm{~B}$ and $\mathrm{D})$, whereas FN mRNA expression levels were not influenced by HOXB9 ( $\mathrm{P}>0.05$; Fig. 2C).

HOXB9 contributes to gel contraction caused by FBs. FBs contract the surrounding matrix, thereby contracting the gel. Compared with healthy skin, hypertrophic scar tissues are characterized by stronger contraction ability. Given that 
$\mathrm{H}$
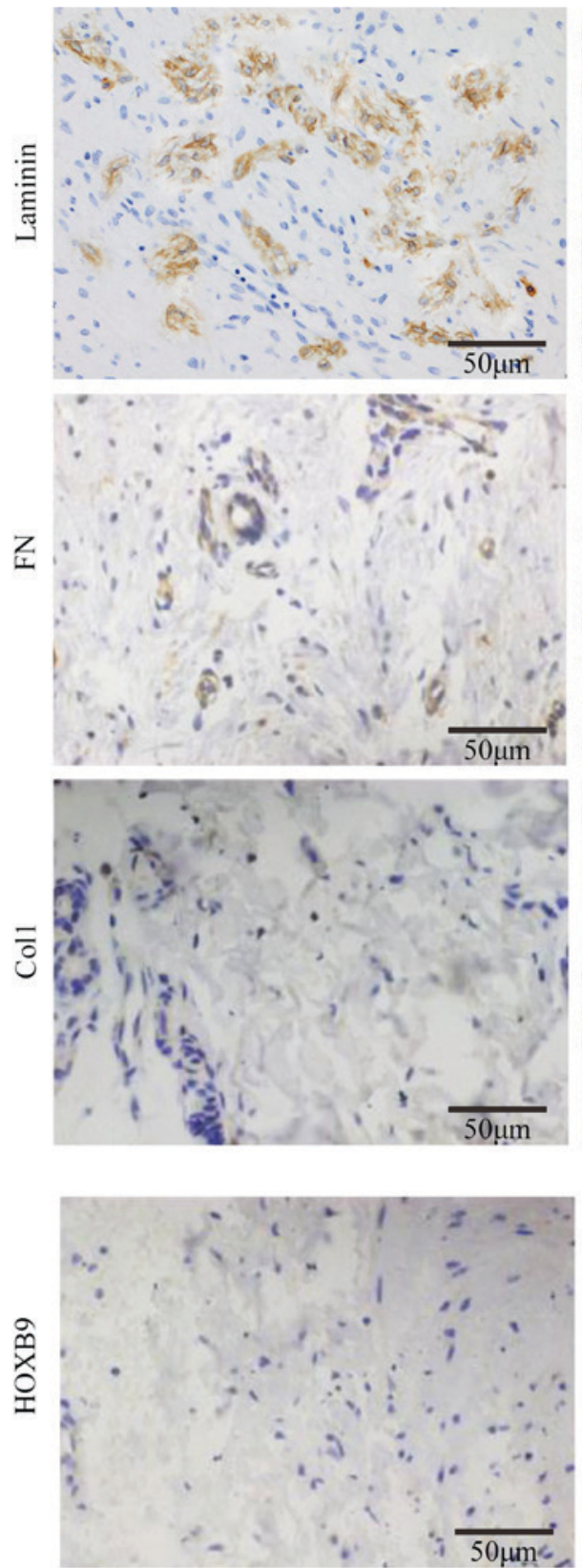

HS
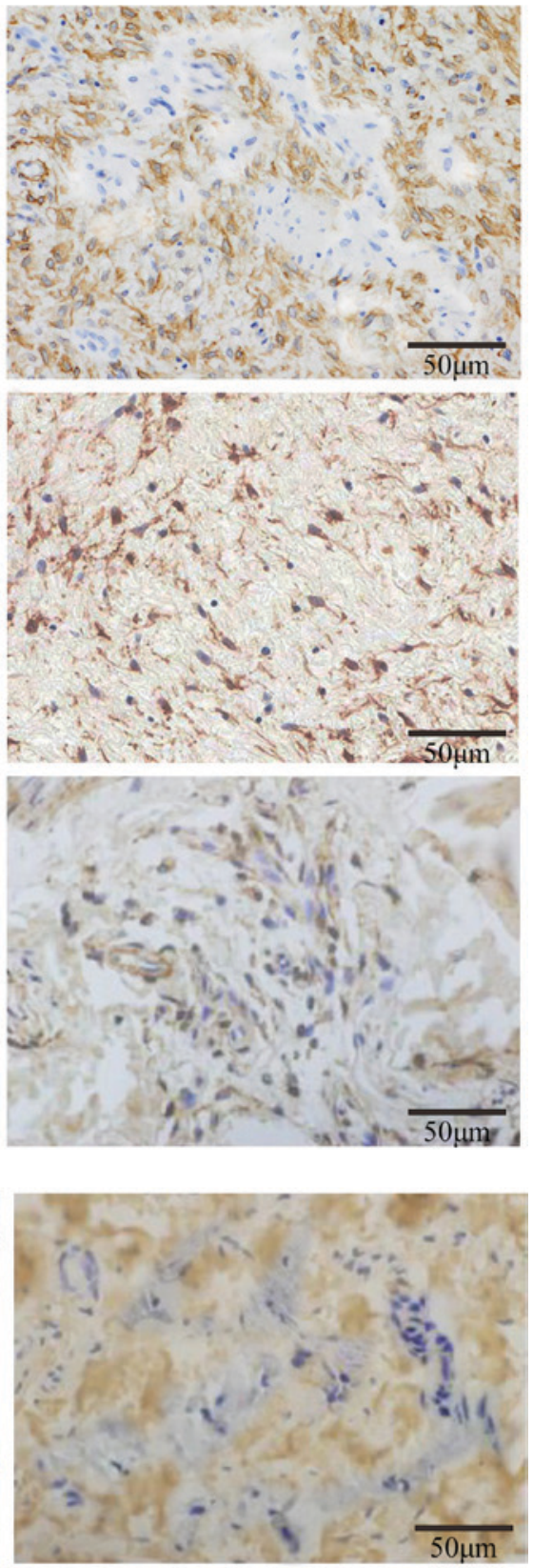

Figure 1. Expression levels of laminin, FN, Col1 and HOXB9 are increased in HS tissue. Scar and healthy adjacent tissues were acquired from patients prior to surgical treatment. Expression of laminin, FN, Coll and HOXB9 were significantly increased in HS tissue compared with autologous normal skin. Scale bar $=50 \mu \mathrm{m}$. HS, hypertrophic scar; FN, fibronectin; Col1, collagen type I; HOXB9, homeobox B9; H, healthy scar tissue.

HOXB9 is highly expressed in hypertrophic scar tissues, investigating the potential roles of HOXB9 in the formation of hypertrophic scars may be significant. To demonstrate whether HOXB9 contributes to gel contraction, FB-mock, FB-negative, FB-HOXB9si and FB-HOXB9over cells were seeded into gels and the gel size was measured 0,24 and $48 \mathrm{~h}$ after seeding. The results clearly demonstrated that $\mathrm{HOXOB9}$ contributes to the gel contraction caused by FBs; the FB-HOXB9over group had a significantly increased gel contraction area at 24 and $28 \mathrm{~h}$ compared with the FB-HOXB9si group ( $\mathrm{P}<0.01$, Fig. 3). Knocking down HOXB9 may attenuate the contraction ability, whereas overexpressing HOXB9 enhances this ability, which is consistent with the results that HOXB9 is highly expressed in hypertrophic scar tissues.
HOXB9 mediates activation of the mitogen-activated protein kinase (MAPK) pathway. MAPK cascades have been demonstrated to serve crucial roles in transmitting extracellular signals to cellular response, leading to an appropriate physiological response including cellular proliferation, differentiation, development, inflammatory responses and apoptosis in mammalian cells. A total of three MAPK families have been characterized, namely classical MAPK (additionally known as ERK), c-Jun JNK and p38 kinase. Given that the production of laminin, FN and Coll are initiated by their corresponding genes activated predominantly by the MAPK signaling pathway, whether HOXB9 mediates MAPK activation was detected. The results clearly demonstrated that knocking down HOXB9 decreased p-ERK, p-JNK and p-p38 production by $40.12 \pm 5.79$ (Fig. 4A), 

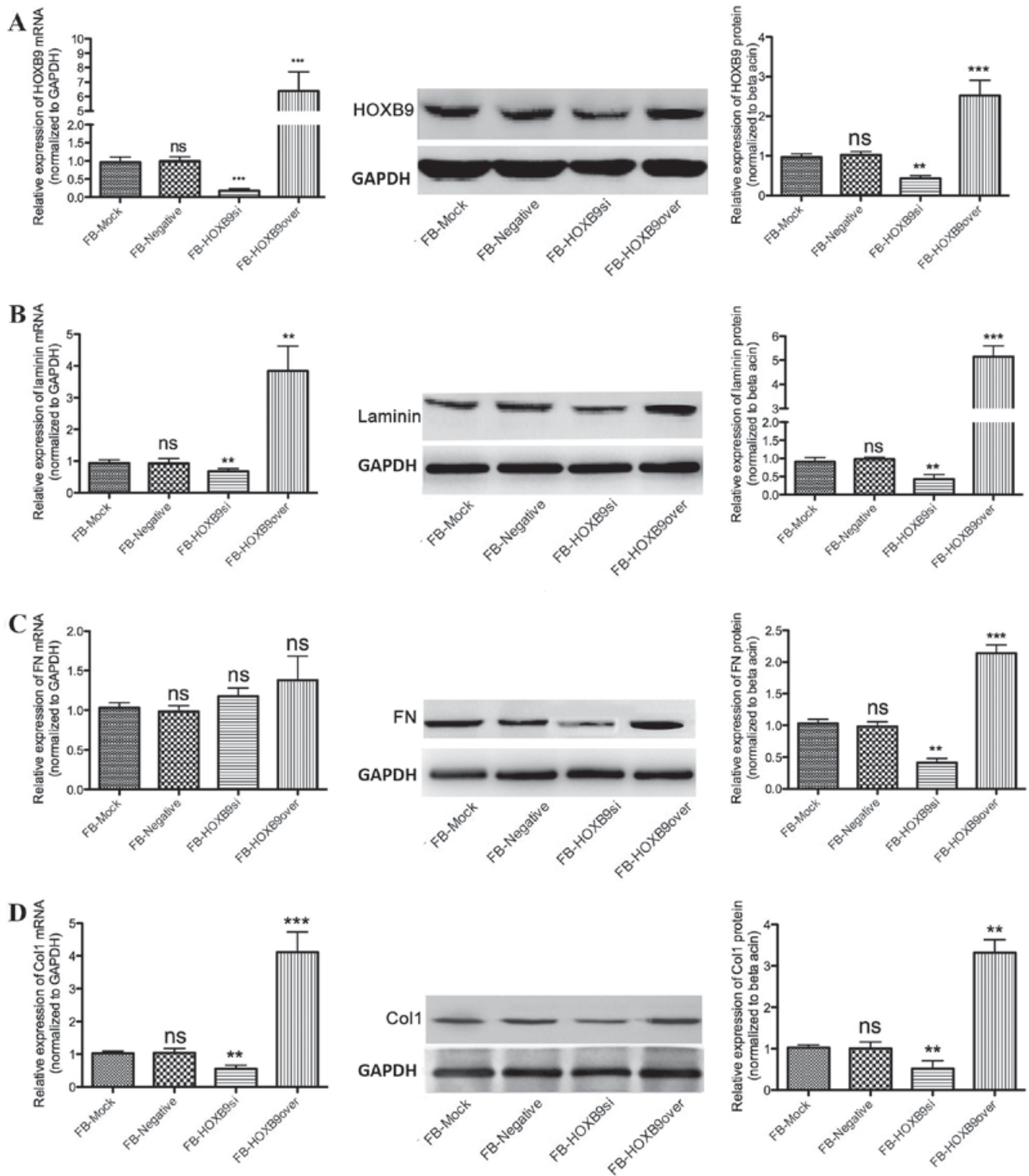

Figure 2. HOXB9 regulates the expression of laminin, FN and Col1 in FBs. Reverse transcription-quantitative polymerase chain reaction and western blotting analyses were performed to assess differential mRNA and protein expression levels, respectively, in FB mock, FB-negative, FB-HOXB9si and FB-HOXB9over cells. (A) FBs were constructed to stably overexpress (FB-HOXB9over) or silence (FB-HOXB9si) HOXB9 using lentiviruses (P<0.001). (B) mRNA and protein expression levels of laminin were decreased in FB-HOXB9si cells and increased in FB-HOXB9over cells, compared with the FB-mock and FB-negative groups $(\mathrm{P}<0.01)$. (C) FN and protein expression levels were decreased in FB-HOXB9si cells and increased in FB-HOXB9over cells, compared with the FB-mock and FB-negative groups $(\mathrm{P}<0.01)$; however, no significant differences were observed in mRNA expression levels $(\mathrm{P}>0.05)$. (D) Col1 mRNA and protein expression levels were decreased in FB-HOXB9si cells and increased in FB-HOXB9over cells, compared with FB-mock and FB-negative cells $(\mathrm{P}<0.01)$. Data are presented as the mean \pm standard deviation of three independent experiments. ${ }^{* *} \mathrm{P}<0.01$ and ${ }^{* * * *} \mathrm{P}<0.001$ vs. FB-mock and FB-negative. FB, fibroblast; FN, fibronectin; HOXB9, homeobox B9; si, silenced; over, overexpressing; Col1; collagen type I; ns, non-significant.

$68.37 \pm 8.72$ (Fig. 4B) and 51.25 $\pm 4.76 \%$ (Fig. 4C), respectively, compared with the FB-mock group $(\mathrm{P}<0.05)$. In contrast, overexpressing HOXB9 increased p-ERK, p-JNK and p-p38 production by $70.33 \pm 9.21 \%$ (Fig. 4A), $69.32 \pm 4.93 \%$ (Fig. 4B) and $1.8 \pm 0.42$ fold (Fig. 4C), respectively, compared with the FB-mock group $(\mathrm{P}<0.01)$.

HOXB9 interacts directly with $p 38$. To further understand the underlying mechanisms by which HOXB9 mediates the MAPK signaling pathway, co-IP experiments were performed using FB extracts to confirm whether HOXB9 interacts with ERK (Fig. 5A), JNK (Fig. 5B) and p38 (Fig. 5C). All three molecules were demonstrated to co-precipitate with HOXB9. However, the reciprocal co-IP experiment revealed that only p38 was present in the HOXB9 precipitate (Fig. 5D). These results confirmed that HOXB9 interacts with p38 in FB cells. It is unclear why the interaction between HOXB9, and ERK and JNK, was inefficient; it may be influenced by temperature and buffer conditions, posttranslational modifications, additional cellular factors and/or the presence of the linker region in ERK and JNK.

\section{Discussion}

The current study aimed to investigate the role of HOXB9 in hypertropic scar formation and the underlying mechanisms. HOXB9 was demonstrated as highly expressed in hypertrophic 


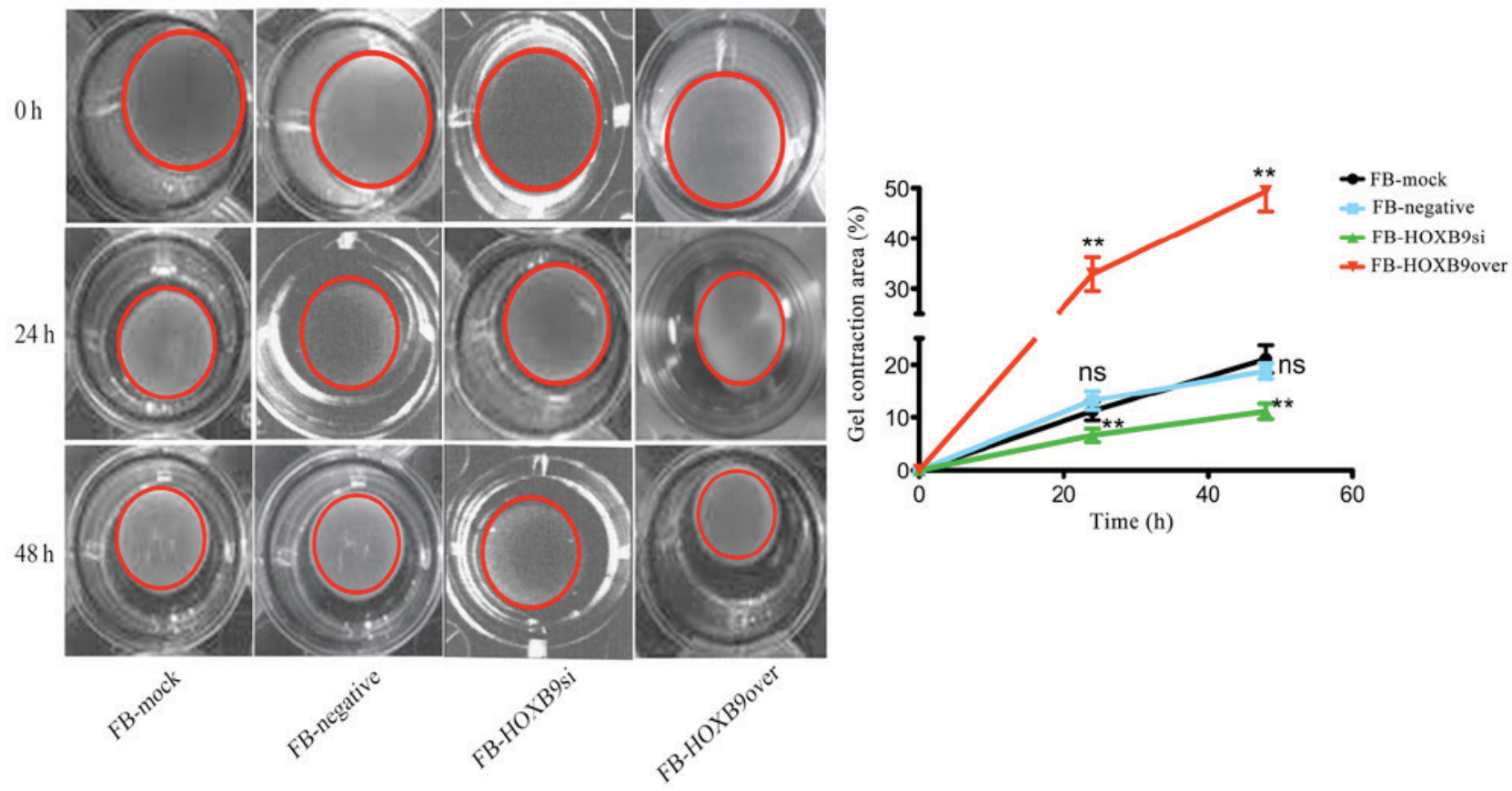

Figure 3. HOXB9 contributes to the contraction of FBs. To assess the contraction function of FB-mock, FB-negative, FB-HOXB9si and FB-HOXB9over cells, the gel area was measured 0,24 and $48 \mathrm{~h}$ after cultivation. The results demonstrated that overexpressing HOXB9 facilitated FB contractions, whereas knocking down HOXB9 attenuated the contractions $(\mathrm{P}<0.01)$. Data are presented as the mean \pm standard deviation of three independent experiments. ${ }^{* *} \mathrm{P}<0.01$ vs. FB-negative and FB-mock groups. FB, fibroblast; HOXB9, homeobox B9; si, silenced; over, overexpressing.
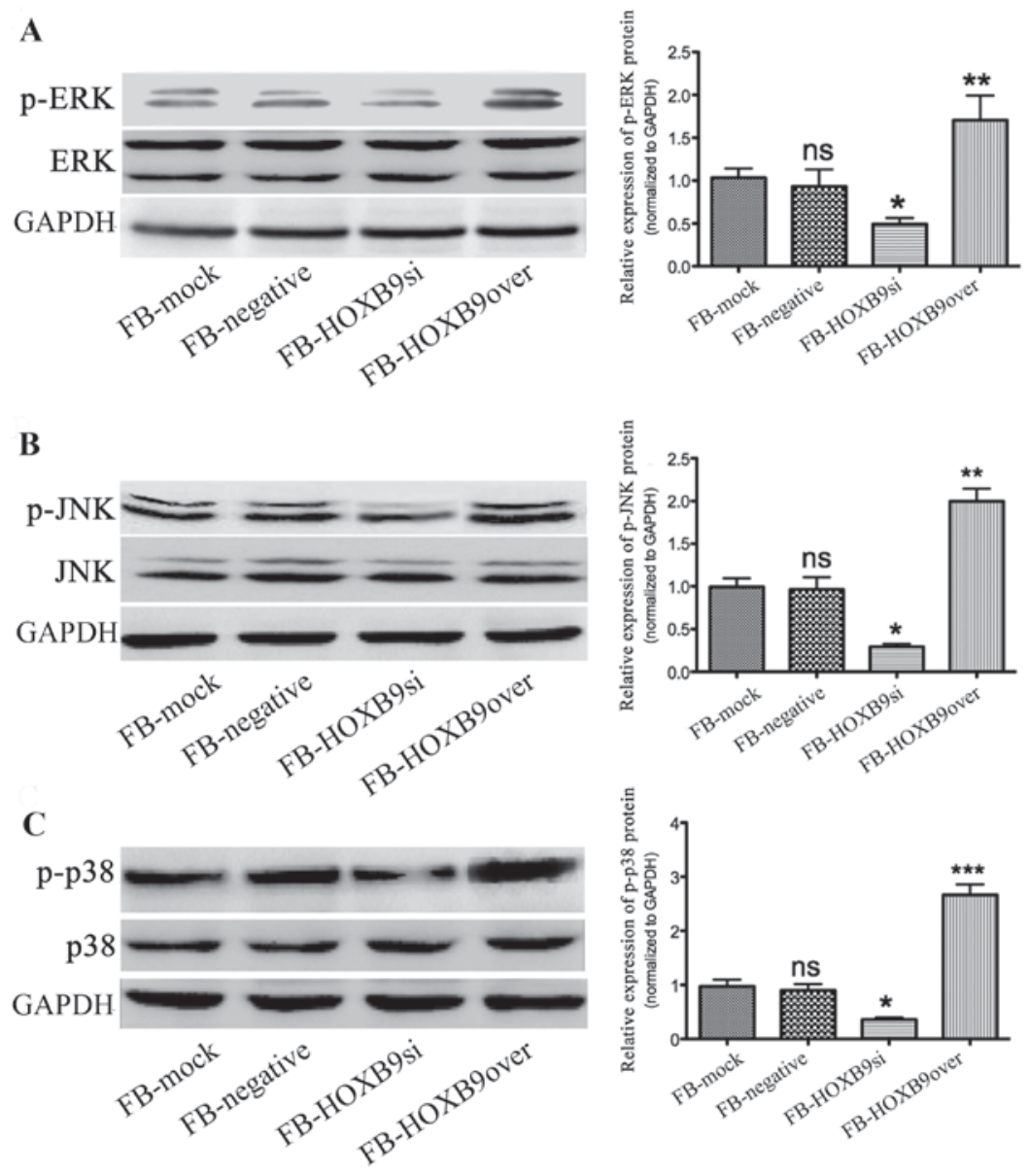

Figure 4. HOXB9 activates the mitogen-activated protein kinase signaling pathway via phosphorylating ERK, JNK and p38. Representative western blot images and quantification of protein expression levels of (A) ERK and p-ERK, (B) JNK and p-JNK, and (C) p38 and P-38. Data are presented as the mean \pm standard deviation of three independent experiments. "P<0.05 and ${ }^{* *} \mathrm{P}<0.01$; ${ }^{* * *} \mathrm{P}<0.001$ vs. FB-mock and FB-negative. FB, fibroblast; HOXB9, homeobox B9; si, silenced; over, overexpressing; p, phosphorylated; ERK, extracellular signal-regulated kinase; JNK, c-Jun N-terminal kinase; ns, non-significant. 
A

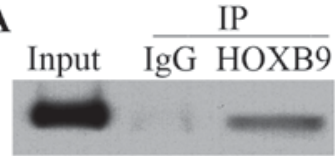

Western blot: anti ERK

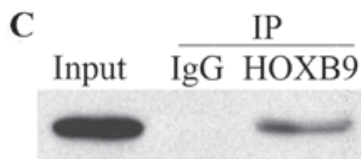

Western blot: anti p38
B

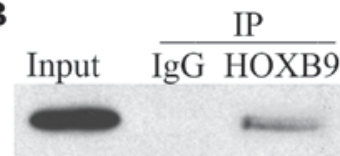

Western blot: anti JNK

D

Western blot: anti HOXB9

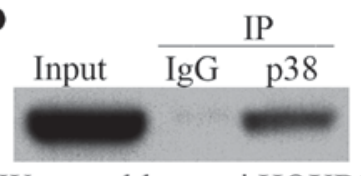

Figure 5. HOXB9 interacts directly with p38. Co-IP was employed to examine whether HOXB9 interacts directly with (A) ERK, (B) JNK and (C) p38. (D) Reverse co-IP confirmed the interaction between HOXB9 and p38, but not ERK or JNK. IP, immunoprecipitation; HOXB9, homeobox B9; ERK, extracellular signal-regulated kinase; JNK, c-Jun N-terminal kinase; ns, non-significant.

scar tissues, accompanied by high expression levels of laminin, FN and Coll. To further examine the potential roles of HOXB9 in hypertrophic scar formation, FB-HOXB9si and FB-HOXB9over vectors were constructed to silence or overexpress the HOXB9 gene, respectively. Western blotting demonstrated that overexpressing HOXB9 elevated laminin, FN and Col1 protein expression, while knocking down HOXB9 decreased their expression. Gel contraction assay supported these findings; increased and reduced HOXB9 expression resulted in enhanced and reduced contraction ability of FBs, respectively. HOXB9 elevated the levels of phosphorylated ERK, JNK and p38, and co-IP revealed an interaction between HOXB9 and p38. To the best of the authors' knowledge, this is the first study to investigate the association between HOXB9 and hypertrophic scar formation, and the underlying mechanisms.

Hypertrophic scars develop from deep dermis injury, including burn injury, surgery and piercings. The annual incidence is $\sim 100$ million patients in the developed world alone each year (33). Hypertrophic scars may greatly decrease the quality of life of the patient, both physically and psychologically, by causing pruritus, pain and contractures. Elaborate efforts have been made to understand the underlying mechanisms of hypertrophic scar formation; however, no satisfactory therapeutic approaches are currently available for this type of lesion.

The ECM consists of structural proteins, collagens, laminins, elastins and FNs to provide flexibility. Abnormal reconstruction of the ECM during wound healing contributes to the formation of hypertrophic scars. The present study collected hypertrophic scar samples from six adults and demonstrated that laminin, $\mathrm{FN}$ and Col1 are highly expressed in hypertrophic scar tissues, which is consistent with previous studies (34).

Previous studies have revealed an association between the HOX genes and wound healing; however, to the best of the authors' knowledge, there are no studies demonstrating the potential association between HOX genes with hypertrophic scar formation. The present study revealed that HOXB9 is highly expressed in hypertrophic scar tissues, implying a potential role in facilitating the hypertrophic scar. To investigate the underlying mechanisms by which HOXB9 may benefit hypertrophic scar formation, FN-HOXB9si and FN-HOXB9over

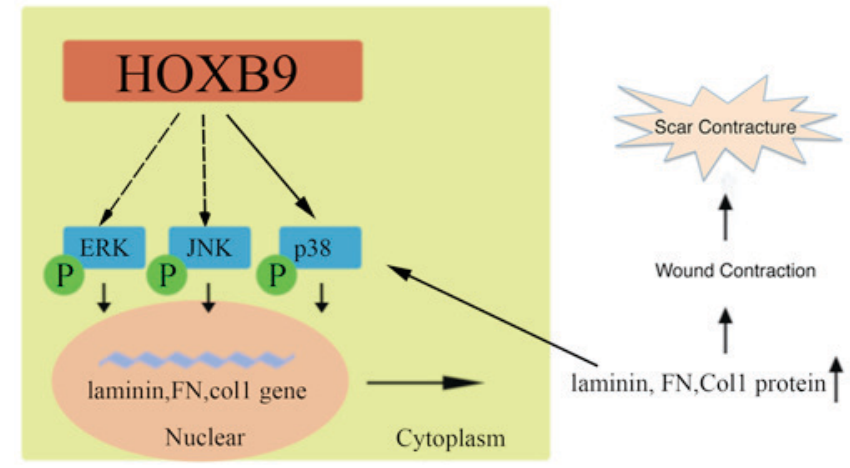

Figure 6. Schematic diagram of HOXB9 facilitating the formation of a hypertrophic scar. HOXB9 activates the MAPK pathway by phosphorylating ERK, JNK and p38, potentially by interacting directly with p38. Activated MAPK leads to the transcription of laminin, FN and Coll mRNA, increasing their protein expression levels, which reconstructs the ECM and contributes to hypertrophic scar formation. Increased laminin, FN and Coll may in return increase p-ERK, p-JNK and p-p38 levels. FN, fibronectin; Col1, collagen type I; HOXB9, homeobox B9; ERK, extracellular signal-regulated kinase; JNK, c-Jun N-terminal kinase; p, phosphorylated; MAPK, mitogen-activated protein kinase.

stable cells were constructed. Knocking down HOXB9 markedly decreased laminin, FN and Coll protein expression levels, while overexpressing HOXB9 elevated their expression. This effect was consistent with the results of the clinical samples, indicating that HOXB9 is involved in the remodeling of the ECM, which is crucial for hypertrophic scar formation. In order to further validate these results, a gel contraction assay was performed to investigate HOXB9 function in hypertrophic scar formation. Gel contraction in the FN-HOXBsi group was significantly decreased, whereas overexpressing HOXB9 in FBs enhanced the contraction ability and greatly increased gel contraction, supporting the hypothesis that HOXB9 facilitates hypertrophic scar formation.

The underlying mechanisms by which HOXB9 upregulates the expression of laminin, FN and Coll were investigated. Increasing evidence has indicated that the MAPK signaling pathway serves an important role in reconstruction of the ECM (35-37). These results demonstrated that knocking down HOXB9 in FBs decreased levels of p-ERK, p-JNK and p-p38, which belong to the MAPK family; whereas overexpressing HOXB9 increased their expression levels, implying that HOXB9 activates the MAPK signaling pathway (38). Co-IP assays demonstrated that HOXB9 interacts directly with ERK, JNK and p38; however, reciprocal co-IP indicated that only p38 interacts with HOXB9. This interaction may lead to accumulation of p-p38, p-ERK and p-JNK, activating MAPK and subsequently elevating the expression levels of laminin, FN and Col1, which results in reconstruction of the ECM and facilitates hypertrophic scar formation. As the MAPK signaling pathways relay, amplify and integrate signals from a wide range of stimuli from the ECM including cytokines, collagen and fibronectin (39), it is possible that these elevated ECM components in return strengthened the activated MAPK signaling pathways (Fig. 6).

Notably, no reciprocal interactions were observed between HOXB9, ERK and JNK, although HOXB9 upregulated p-ERK and p-JNK levels, suggesting that additionally underlying mechanisms by which HOXB9 may induces the phosphorylation of ERK and JNK should be investigated. Animal models 
used for scar research should additionally be examined to further verify these findings.

In conclusion, the present study demonstrated that HOXB9 effectively facilitated hypertrophic scar formation and strengthened the contractile ability of FBs via activating the MAPK signaling pathway in vivo. HOXB9 may serve as a potential therapeutic target for the treatment of hypertrophic scars or other fibroproliferative disorders, and requires further investigation.

\section{Acknowledgements}

The authors would like to thank Professor Shengguo Shan (Renmin Hospital of Wuhan University, Wuhan, China) for his assistance in preparing the manuscript.

\section{References}

1. Rabello FB, Souza CD and Farina Júnior JA: Update on hypertrophic scar treatment. Clinics (Sao Paulo) 69: 565-573, 2014.

2. Branski LK, Rennekampff HO and Vogt PM: Keloid and hypertrophic scar treatment modalities. An update. Chirurg 83 831-846, 2012 (In German).

3. Hayashi T, Furukawa H, Oyama A, Funayama E, Saito A, Murao N and Yamamoto Y: A new uniform protocol of combined corticosteroid injections and ointment application reduces recurrence rates after surgical keloid/hypertrophic scar excision. Dermatol Surg 38: 893-897, 2012.

4. Huang D, Shen KH and Wang HG: Pressure therapy upregulates matrix metalloproteinase expression and downregulates collagen expression in hypertrophic scar tissue. Chin Med J (Engl) 126: 3321-3324, 2013

5. Chesnut C, Mednik S and Lask G: Hypertrophic scar treatment with intralesional triamcinolone acetonide and pulsed dye laser results in necrosis. Cutis 94: E12-E13, 2014.

6. On HR, Lee SH, Lee YS, Chang HS, Park C and Roh MR: Evaluating hypertrophic thyroidectomy scar outcomes after treatment with triamcinolone injections and copper bromide laser therapy. Lasers Surg Med 47: 479-484, 2015.

7. Al-Mohamady Ael-S, Ibrahim SM and Muhammad MM: Pulsed dye laser versus long pulsed Nd:YAG laser in the treatment of hypertrophic scars and keloid: A comparative randomized split-scar trial. J Cosmet Laser Ther 18: 208-212, 2016.

8. Zhu Z, Ding J, Shankowsky HA and Tredget EE: The molecular mechanism of hypertrophic scar. J Cell Commun Signal 7 239-252, 2013.

9. Werner S, Krieg T and Smola H: Keratinocyte-fibroblast interactions in wound healing. J Invest Dermatol 127: 998-1008, 2007.

10. Li B and Wang JH: Fibroblasts and myofibroblasts in wound healing: Force generation and measurement. J Tissue Viability 20: 108-120, 2011.

11. Gehring WJ, Affolter M and Bürglin T: Homeodomain proteins. Annu Rev Biochem 63: 487-526, 1994.

12. Apiou F, Flagiello D, Cillo C, Malfoy B, Poupon MF and Dutrillaux B: Fine mapping of human HOX gene clusters. Cytogenet Cell Genet 73: 114-115, 1996.

13. Mace KA, Hansen SL, Myers C, Young DM and Boudreau N: HOXA3 induces cell migration in endothelial and epithelial cells promoting angiogenesis and wound repair. J Cell Sci 118 2567-2577, 2005.

14. Hansen SL, Myers CA, Charboneau A, Young DM and Boudreau N: HoxD3 accelerates wound healing in diabetic mice. Am J Pathol 163: 2421-2431, 2003.

15. Mack JA and Maytin EV: Persistent inflammation and angiogenesis during wound healing in K14-directed Hoxb13 transgenic mice. J Invest Dermatol 130: 856-865, 2010.

16. Mack JA, Abramson SR, Ben Y, Coffin JC, Rothrock JK, Maytin EV, Hascall VC, Largman C and Stelnicki EJ: Hoxb13 knockout adult skin exhibits high levels of hyaluronan and enhanced wound healing. FASEB J 17: 1352-1354, 2003.

17. Chen F and Capecchi MR: Paralogous mouse Hox genes, Hoxa9, Hoxb9 and Hoxd9, function together to control development of the mammary gland in response to pregnancy. Proc Natl Acad Sci USA 96: 541-546, 1999.
18. Seki H, Hayashida T, Jinno H, Hirose S, Sakata M, Takahashi M, Maheswaran S, Mukai M and Kitagawa Y: HOXB9 expression promoting tumor cell proliferation and angiogenesis is associated with clinical outcomes in breast cancer patients. Ann Surg Oncol 19: 1831-1840, 2012.

19. Kwon OS, Oh E, Park JR, Lee JS, Bae GY, Koo JH, Kim H, Choi YL, Choi YS, Kim J and Cha HJ: GalNAc-T14 promotes metastasis through Wnt dependent HOXB9 expression in lung adenocarcinoma. Oncotarget 6: 41916-41928, 2015.

20. Zhan J, Wang P, Niu M, Wang Y,Zhu X, Guo Y and Zhang H: High expression of transcriptional factor HoxB9 predicts poor prognosis in patients with lung adenocarcinoma. Histopathology 66 : 955-965, 2015.

21. Hayashida T, Takahashi F, Chiba N, Brachtel E, Takahashi M, Godin-Heymann N, Gross KW, Vivanco Md, Wijendran V, Shioda T, et al: HOXB9, a gene overexpressed in breast cancer, promotes tumorigenicity and lung metastasis. Proc Natl Acad Sci USA 107: 1100-1105, 2010

22. Nguyen DX, Chiang AC, Zhang XH, Kim JY, Kris MG, Ladanyi M, Gerald WL and Massagué J: WNT/TCF signaling through LEF1 and HOXB9 mediates lung adenocarcinoma metastasis. Cell 138: 51-62, 2009.

23. Sha S, Gu Y, Xu B, Hu H, Yang Y, Kong X and Wu K: Decreased expression of HOXB9 is related to poor overall survival in patients with gastric carcinoma. Dig Liver Dis 45: 422-429, 2013.

24. Chang Q, Zhang L, He C, Zhang B, Zhang J, Liu B, Zeng N and Zhu Z: HOXB9 induction of mesenchymal-to-epithelial transition in gastric carcinoma is negatively regulated by its hexapeptide motif. Oncotarget 6: 42838-42853, 2015.

25. He T, Bai X, Yang L, Fan L, Li Y, Su L, Gao J, Han S and Hu D: Loureirin B inhibits hypertrophic scar formation via inhibition of the TGF- $\beta 1$-ERK/JNK Pathway. Cell Physiol Biochem 37: 666-676, 2015.

26. Root DE, Hacohen N, Hahn WC, Lander ES and Sabatini DM: Genome-scale loss-of-function screening with a lentiviral RNAi library. Nat Methods 3: 715-719, 2006

27. Livak KJ and Schmittgen TD: Analysis of relative gene expression data using real-time quantitative PCR and the 2(-Delta Delta C(T)) method. Methods 25: 402-408, 2001.

28. Ngo P, Ramalingam P, Phillips JA and Furuta GT: Collagen gel contraction assay. Methods Mol Biol 341: 103-109, 2006.

29. Vernon RB and Gooden MD: An improved method for the collagen gel contraction assay. In Vitro Cell Dev Biol Anim 38: 97-101, 2002.

30. Viera MH, Amini S, Valins $\mathrm{W}$ and Berman B: Innovative therapies in the treatment of keloids and hypertrophic scars. J Clin Aesthet Dermatol 3: 20-26, 2010.

31. Shaarawy E, Hegazy RA and Abdel Hay RM: Intralesional botulinum toxin type A equally effective and better tolerated than intralesional steroid in the treatment of keloids: A randomized controlled trial. J Cosmet Dermatol 14: 161-166, 2015.

32. Gauglitz GG, Bureik D, Dombrowski Y, Pavicic T, Ruzicka T and Schauber J: Botulinum toxin A for the treatment of keloids. Skin Pharmacol Physiol 25: 313-318, 2012.

33. Gauglitz GG, Korting HC, Pavicic T, Ruzicka T and Jeschke MG: Hypertrophic scarring and keloids: Pathomechanisms and current and emerging treatment strategies. Mol Med 17: 113-125, 2011

34. Xue $M$ and Jackson CJ: Extracellular matrix reorganization during wound healing and its impact on abnormal scarring. Adv Wound Care (New Rochelle) 4: 119-136, 2015.

35. Crean JK, Finlay D, Murphy M, Moss C, Godson C, Martin F and Brady HR: The role of p42/44 MAPK and protein kinase $\mathrm{B}$ in connective tissue growth factor induced extracellular matrix protein production, cell migration, and actin cytoskeletal rearrangement in human mesangial cells. J Biol Chem 277: 44187-44194, 2002.

36. Xiao G, Gopalakrishnan R, Jiang D, Reith E, Benson MD and Franceschi RT: Bone morphogenetic proteins, extracellular matrix, and mitogen-activated protein kinase signaling pathways are required for osteoblast-specific gene expression and differentiation in MC3T3-E1 cells. J Bone Miner Res 17: 101-110, 2002.

37. Qin H, Ishiwata T, Wang R, Kudo M, Yokoyama M, Naito Z and Asano G: Effects of extracellular matrix on phenotype modulation and MAPK transduction of rat aortic smooth muscle cells in vitro. Exp Mol Pathol 69: 79-90, 2000.

38. Kyriakis JM and Avruch J: Mammalian MAPK signal transduction pathways activated by stress and inflammation: A 10 -year update. Physiol Rev 92: 689-737, 2012.

39. Zhang W and Liu HT: MAPK signal pathways in the regulation of cell proliferation in mammalian cells. Cell Res 12: 9-18, 2002. 